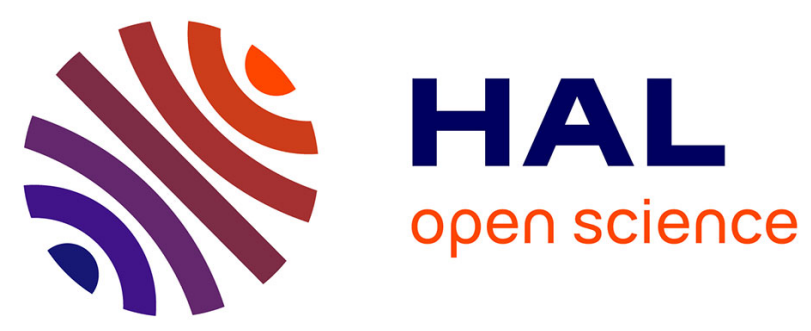

\title{
Experimental investigation of holes interaction effect on the sound absorption coefficient of micro-perforated panels under high and medium sound levels
}

Rostand Tayong, Thomas Dupont, Philippe Leclaire

\section{To cite this version:}

Rostand Tayong, Thomas Dupont, Philippe Leclaire. Experimental investigation of holes interaction effect on the sound absorption coefficient of micro-perforated panels under high and medium sound levels. Applied Acoustics, 2011, 72, pp.777-784. 10.1016/j.apacoust.2011.04.011 . hal-01331522

HAL Id: hal-01331522

https://hal.science/hal-01331522

Submitted on 13 Jun 2016

HAL is a multi-disciplinary open access archive for the deposit and dissemination of scientific research documents, whether they are published or not. The documents may come from teaching and research institutions in France or abroad, or from public or private research centers.
L'archive ouverte pluridisciplinaire HAL, est destinée au dépôt et à la diffusion de documents scientifiques de niveau recherche, publiés ou non, émanant des établissements d'enseignement et de recherche français ou étrangers, des laboratoires publics ou privés.

\section{다)(1) $\$$}

Distributed under a Creative Commons Attribution - NonCommerciall 4.0 International 


\title{
Experimental investigation of holes interaction effect on the sound absorption coefficient of micro-perforated panels under high and medium sound levels
}

\author{
Rostand Tayong*, Thomas Dupont, Philippe Leclaire \\ Laboratoire de Recherche en Mécanique et Acoustique (DRIVE-LRMA), Université de Bourgogne, ISAT, 49 Rue Mademoiselle Bourgeois, 58027 Nevers, France
}

\begin{abstract}
A B S T R A C T
i nis paper experımentany investigates tne noies interaction eirect on tne sound absorpuon coemcient or micro-perforated panels under high and medium sound levels. The theoretical formulations are based on a semi-empirical approach and the use of Fok's function to model the acoustic surface impedance. For the high sound level regime, an empirical power law involving three coefficients is adapted. It is shown theoretically and experimentally that these coefficients can lead to optimized absorption performance and particularly, a formula relating the critical Reynolds number (Reynolds number value after which the absorption coefficient decreases with the increase of sound level) and the center-to-center distance between the perforations is derived. It is demonstrated that the first coefficient of the nonlinear acoustic resistance strongly depends on the separation distance between the apertures and decreases with a decrease of this latter distance. Analysis of the data reveals the fact that even with Holes Interaction Effect (HIE), the nonlinear reactance dependence on velocity is still very low compared to the resistancevelocity dependence. Four perforated panels of $1.5 \mathrm{~mm}$ thickness with different separation distances between the holes (from widely to closely separation) were built and tested. Experimental results performed with an impedance tube are compared with the described model for HIE. To test the dependence of the coefficients on frequency, the experiments are carried out for two different excitation frequencies $(292 \mathrm{~Hz}$ and $506 \mathrm{~Hz}$ ). The results can be used for designing optimal perforated panels for ducts, silencers and for the automotive industry.
\end{abstract}

\section{Introduction}

In various noise control applications such as ducts, exhaust systems, automotive and aircraft, perforated panels are used for sound attenuation purposes. One of the advantages of using perforated panels is that their resonant frequencies can be tuned depending on the goal to be achieved. Moreover, they are proved to be robust, easy to manufacture and reliable in hostile temperature and pressure environments. When the perforations are reduced to millimeter or sub-millimeter size (the term microperforated panel is rather used for sub-millimetric radius), these materials present very interesting sound absorption characteristics without any additional classical absorbing material. They are also proved to be very useful in dealing with low-frequency noise.

A large number of models have been proposed for the acoustical behavior of perforated and micro-perforated panels.
One common particularity of most of these models [1-3] is that they are only applicable for widely separated holes (assumption of no interaction between the perforations). However, in practical applications, the orifices are usually closely spaced, such that the interaction between neighboring orifices can strongly influence the acoustic performance of perforated liners. Ingard [4] presented an extensive survey on the topic of resonators. In his work two interacting apertures have been studied and the end correction is shown to be strongly dependent on the separation between the holes. Very recently, Atalla and Sgard [5] derived a model for perforated plates using an approach originally proposed for rigid frame porous materials and applicable only for low sound levels regimes (linear regime). Their model accounts for very weak interaction between the apertures. The commonly accepted approach to Holes Interaction Effect (HIE) is due to Fok [6]. From his work [6,7], a function (now called the Fok's function) was derived taking into account the center-to-center distance between the perforations and the correction for the radiating impedance of interacting perforations. These latter studies were focused on the case of low sound levels also known as the linear regime. In the nonlinear regime (case of high sound pressure levels), Ingard and Ising [8] showed, with the help of a hot wire probe (technique 
of measurement of velocity and temperature fluctuations for investigations in turbulent flows), that at high sound pressure levels, the acoustic particle velocity is increased sharply as the wave is squeezed into the minute perforation and the flow separates at the outlet orifice, forming a high velocity jet. During the inflow half-cycle, the flow at the inlet of the orifice is essentially irrotational but becomes highly rotational (in form of jetting) after exiting from the outlet orifice. Cummings $[9,10]$ works on a perforated plate lead to the development of a one-dimensional numerical time-domain model to simulate the nonlinear resistance of such a plate assuming no holes interaction. Later on, also assuming no interaction between the apertures, Kraft [11] proposed a model for micro-perforated panel behavior at high levels of excitation taking into account the discharge coefficient. His model was derived from measurements of single degree-of-freedom liners over a wide range of sound pressure levels. The model provided a prediction for the combined linear and nonlinear resistance and reactance of a face sheet. The first study of perforated plates in the nonlinear regime accounting for HIE may be related to Melling [12] whose study was focused on the cases of $22 \%$ and $7.5 \%$ porosities. He made the use of Fok's function and derived a model using the discharge coefficient (ratio of the actual discharge divided by the ideal discharge). Moreover he noted that in practice the radiating impedance correction of Fok will be small for low porosity (known as the open area ratio for perforated plates) samples; high porosity samples will reduce the end-correction by a significant amount. Under high sound pressure levels, the information about holes interaction is implicitly contained in the discharge coefficient values. Later, Randeberg [13] revealed specifically that in the linear regime, the result of applying Fok's function as a correction due to the interaction between the holes is practically equivalent to using Ingard's inner end correction for both apertures of holes in a perforated panel. By solving the Euler's equation for incompressible fluid for an orifice in a finitethickness partition that spans a tube, Lee et al. [14] recently analyzed numerically and experimentally a model for the acoustic impedance of perforated plates in the presence of a flow. In their work, the HIE was examined only on the acoustic impedance and transmission loss of perforated baffles. They presented simulations and experiments that showed reasonable agreement. Nevertheless, there is still a great need for studies dealing specifically with HIE for perforated panels subjected to high sound pressure levels since all the phenomena involved are not quite mastered yet.

This paper is an extended version of a talk presented at the 10th French Congress of Acoustics in Lyon [15]. The objective of this paper is to experimentally investigate the HIE on the sound absorption of perforated or micro-perforated panels when backed by an air cavity under medium and high sound pressure levels. The study does not take into account the presence of mean flow. Since perforated plates are proved to be useful in dealing with low-frequency noise, the choice of two different relatively low frequencies $(292 \mathrm{~Hz}$ and $506 \mathrm{~Hz}$ ) is made to test the dependence of the nonlinear coefficients on frequency. As a first step, Fok's function accounting for the holes interaction is presented. Next, Ingard's classical model is described and adapted for high sound pressure levels with the use of an empirical power law. In the power law expression, two adjustable coefficients are defined to fit the data at high sound pressure levels. The experimental setup is then described. Very carefully controlled measurements are carried out on samples with different separation distances between the holes (from widely separated to closely separated holes). The effect of holes interaction on the resistance, the reactance and the maximum of absorption coefficient is particularly discussed. The results are summarized in the conclusion of this paper.

\section{Theoretical formulations}

\subsection{Holes interaction function: Fok's function}

In this study, the acoustic wavelengths are considered to be much larger than the plate dimensions (thickness and hole diameter) and the radiation of sound from an aperture is considered to be spherical. Assuming equal radiation in all directions, the wave equation in spherical coordinates with no angle dependence (see Ref. [16]) is written as

$\frac{\partial^{2} p}{\partial r^{2}}+\frac{2}{r} \frac{\partial p}{\partial r}=\frac{1}{c_{0}^{2}} \frac{\partial^{2} p}{\partial t^{2}}$

where $p$ is the sound pressure and $c_{0}$ is the sound speed in air. Under the latter assumption, spherical sound waves will travel away from a single aperture and the wave front will remain spherical. At the outlet of the aperture, the flow field can be studied in terms of pressure, velocity or even shear.

Consider the case of 2 apertures of the same area $\left(S_{1}=S_{2}=\mathrm{S}\right)$ positioned closely to each other. If the pressure field radiating from the first aperture is denoted $p_{1}$ and the pressure field radiating from the second aperture is $p_{2}$, the total pressure is $p_{1}+p_{2}$. The impedance $z_{1,2}$ due to the interaction between the apertures can be obtained by calculating the pressure on the surface of each opening caused by the radiation from the neighboring one and given by Ingard [4] as

$z_{1,2}=\frac{1}{U_{2} S_{2}} \int_{S_{2}} p_{1,2} d S_{2}$

where $p_{1,2}$ is the pressure exerted by aperture 1 at aperture $2, U_{2}$ is the particle velocity through aperture 2. Eq. (2) quantifies the end correction due to the interaction effect [4]. In fact, Ingard [4] shows that

$z_{12}=(-i \omega) \pi r^{2} \rho_{0} \delta_{12}$

where $\omega$ is the angular frequency, $\rho_{0}$ is the density of air, $r$ is the aperture radius and $\delta_{12}$ is the interaction end correction. Now, the rigorous calculations of $\delta{ }_{12}$ for a given geometry is difficult and cannot be done analytically in general case. For purposes of interpretation, this end correction must be expressed as a function of $d / b$ (ratio of hole diameter $d$ and distance b between the holes centers). The HIE that takes place out of the apertures is linked to the propagation inside the aperture (as noticed by Ingard [4]). One important parameter used to describe the propagation inside the hole is the acoustic conductance (dimension of length [16]) given for low frequencies by

$E_{c}=\frac{1}{2} \frac{\rho_{0}}{K_{r}} Q^{2}$

where $E_{c}$ is the kinetic energy of the air inside the aperture, $K_{r}$ is the conductance and $Q$ is the volume velocity. From Eq. (4), one can notice that $K_{r}$ is inversely proportional to the oscillating mass of air inside the aperture. Following Rayleigh's work [16] on the attached mass of a circular aperture, Fok [6,7] derived an expression for the attached mass $M$ given by

$M=\frac{\rho_{0} s^{2}}{K_{r}}=\frac{\rho_{0} s^{2}}{d \psi}$,

where $s$ is the aperture area and $\psi$ is a polynomial approximation function (also known as Fok's function) expressed as

$\psi(\xi)=\left(1+x_{1} \xi+x_{2} \xi^{2}+x_{3} \xi^{3}+\cdots\right)^{-1}$,

where $\xi=\frac{\sqrt{\pi}}{2} \frac{d}{b}$, with $x_{1}=-1.4092 ; x_{2}=0 ; x_{3}=+0.33818 ; x_{4}=0 ; x_{5}=$ $+0.06793 ; \quad x_{6}=-0.02287 ; \quad x_{7}=+0.03015 ; \quad x_{8}=-0.01641 ; \quad x_{9}=$ $+0.01729 ; x_{10}=-0.01248 ; x_{11}=+0.01250 ; x_{12}=-0.00985$. This latter 
function can be applied to the end correction length. It is worth noticing that Fok's function is derived under the assumption of potential flow conditions. Therefore, when the flow through the hole is not a potential flow (case of high sound level regime where turbulence occurs for instance), the application of Fok's function is quite limited as would be shown by the experimental results.

Fig. 1 presents Fok's function as a function of $d / b$. One can then observe that:

- When $d / b$ tends to unity, the apertures are close to each other and $\xi$ tends to $\frac{\sqrt{\pi}}{2} \approx 0.8$. Therefore, Fok's function value tends to infinity (see Eq. (6)) and the attached mass tends to zero (see Eq. (5)).

- When $d / b<0.2$, the apertures are widely separated from each other, Fok's function value tends to unity (Fig. 1) and the attached mass is equivalent to the one of a single aperture (the conductance being equal to the diameter [16]). In this case, the interaction effect between the aperture is negligible.

2.2. Derivation of the surface impedance and the absorption coefficient accounting for the holes interaction effect

To take into account the interaction effect between the apertures, Rschevkin [7] proposed modifying the end correction term in the model for perforated plate with the Fok's function. Due to the flow of air through the holes which affects the air close to the inner and outer apertures, the thickness must be corrected with an additional term [1]. For circular cross-section holes, Rayleigh [16] proposed an end correction $l_{0}$ for the inner and outer aperture as follows

$l_{0}=\frac{8 d}{3 \pi}$

Following Ingard's work $[4,13]$, the acoustic impedance of a single perforation accounting for the inlet and outlet end effects of the hole is given by

$Z_{1}=4 R_{s}\left(1+\frac{h}{d}\right)+j \omega \rho_{0}\left(h+l_{0}\right)$,

where $h$ is the panel thickness and the surface resistance $R_{s}$ due to viscous dissipation inside the aperture and at the surface of the plate is given by

$R_{s}=\frac{1}{2} \sqrt{2 \omega \rho_{0} \mu}$,

where $\mu$ is the dynamic viscosity of air. Finally, the normalized impedance $z_{l}$ of the perforated panel accounting for interaction between the holes in the linear regime is given by

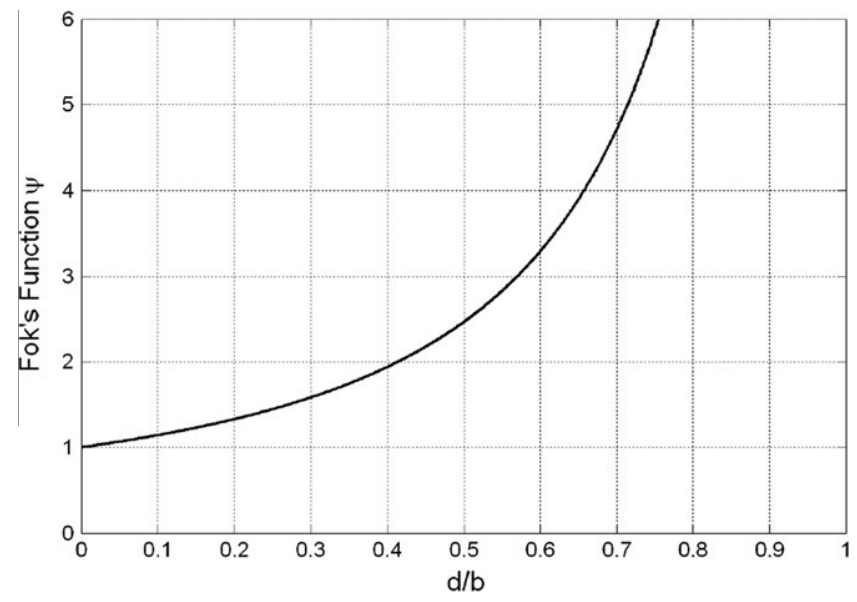

Fig. 1. Fok's function $\Psi(\xi)$ versus $d / b$ (Fig. 6 of Ref. [12]). $z_{l}=\frac{4 R_{s}}{\phi Z_{0}}\left(1+\frac{h}{d}\right)+\frac{j \omega \rho_{0}}{\phi Z_{0}}\left(h+\frac{l_{0}}{\psi(\xi)}\right)$

where $\phi$ is the open area ratio, $Z_{0}$ is the characteristic impedance of air and $\psi(\xi)$ is the Fok's function as described in the previous section. As shown in Fig. 1 (so as in Fig. 6 of Ref. [12]) when $d /$ $b<0.2$, the Fok's function value tends to unity and Eq. (10) becomes equivalent to Eq. (8). Therefore in this case, there is no appreciable interaction effect. When $d / b$ tends to unity, the Fok's function value tends to infinity and the imaginary part (also known as the reactance) of Eq. (10) tends to zero. Therefore in this case, the attached mass is effectively zero as explained earlier.

According to Ingard[4], under high sound pressure levels, assuming that the reactance dependence on velocity is negligible, the equation for the velocity dependent resistance is given by

$\frac{R_{n l}}{d}=K_{1}\left(\frac{U}{100}\right)^{C_{2}}$

where $R_{n l}$ is the nonlinear resistance to be added to the acoustic impedance in the linear regime, $U$ is the average incident velocity, $K_{1}$ and $C_{2}$ are constants that may depend on frequency. For a single frequency and using the Reynolds number, the normalized form of Eq. (11) can be transformed into

$r_{n l}=C_{1} \operatorname{Re}^{C_{2}}$,

where $R e$ is the Reynolds number, $C_{1}$ and $C_{2}$ are adjustable coefficients defined to fit the data. Their values for the samples used for measurements are given in Table 2 . The Reynolds number is expressed as

$\operatorname{Re}=\frac{d U}{v}$

with $v$ the kinematic viscosity of air. As one can notice, only the real part (resistance) of the acoustic impedance is dependent on the incident velocity whereas the imaginary part (reactance) dependence on velocity is assumed negligible. This assumption is generally considered in most models $[11,12]$. If the perforated panel is backed by an air cavity, the resonant system formed has a general normalized impedance given by

$z_{s}=r_{n l}+(1+\delta) z_{l}+z_{c a v}$,

where $z_{c a v}$ is the normalized impedance of the air cavity and $\delta$ is characteristic of three regimes: the linear regime $(\delta=0)$, the weakly nonlinear regime $(\delta \neq 0)$, and a transition regime between them. It should be noted that $\delta$ has no real physical interpretation as for $R e=0$, the asymptotic behavior does not hold (the values for $\delta$ are given in Table 2). The normalized impedance of the air cavity is expressed as

$z_{\text {cav }}=-j \cot \left(k_{0} D_{c}\right)$

$k_{0}$ is the wave number and $D_{c}$ the air cavity depth. The reflection coefficient is obtained using the formula

$R=\frac{z_{s}-1}{z_{s}+1}$

and the absorption coefficient is given by

$\alpha=1-|R|^{2}$.

\section{Experimental setup}

All the measurements were performed on aluminum perforated panels. Each sample has an external diameter of $100 \mathrm{~mm}$. The mounting conditions of the samples inside the tube are close to a clamped condition. Pictures of the samples used in the experiments are shown in Fig. 2 and the samples characteristics are given in Table 1. 

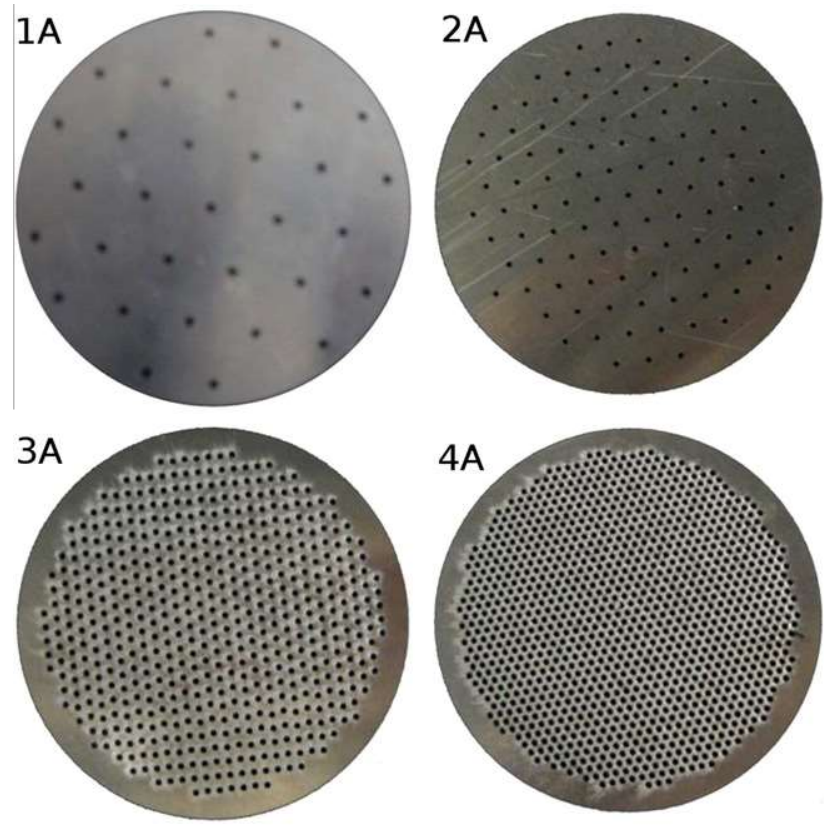

Fig. 2. Sample pictures.

Table 1

Dimensions of the perforated samples.

\begin{tabular}{lllcrl}
\hline & $h(\mathrm{~mm})$ & $d(\mathrm{~mm})$ & $b(\mathrm{~mm})$ & $\phi(\%)$ & $\xi=d / b$ \\
\hline Sample 1A & 1.5 & 1.6 & 12 & 1.92 & 0.13 \\
Sample 2A & 1.5 & 1.6 & 8.0 & 4.25 & 0.2 \\
Sample 3A & 1.5 & 1.6 & 3.5 & 20.52 & 0.45 \\
Sample 4A & 1.5 & 1.6 & 2.6 & 35.28 & 0.61 \\
\hline
\end{tabular}

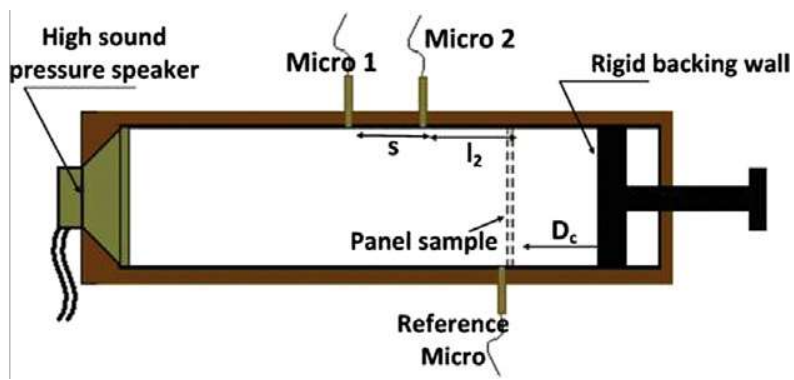

Fig. 3. Schematic of the impedance tube used for the measurements.

A schematic diagram of the impedance tube set-up used for the experiments is shown in Fig. 3. It is a rigid circular plane-wave tube with a diameter of $100 \mathrm{~mm}$ (cut-off frequency of the tube is $1.7 \mathrm{kHz}$ ). On the left hand side, a JBL model $2450 \mathrm{~J}$ compression driver is mounted as the excitation source. This compression driver is powered by a power amplifier. When performing high sound pressure measurements inside an impedance tube, it is important to check the resulting standing wave. Depending on the frequency excitation and the sample characteristics, the standing wave within the impedance tube may contain relatively high nonlinear harmonics. If this occurs, the linear propagation hypothesis is no longer valid. This phenomenon was studied by Maa and Liu [17]. In our study, we made sure that the nonlinear harmonics were sufficiently small so that the impedance tube method was applicable. To insure a smooth transition between the circular section of the compression driver (throat diameter of $49 \mathrm{~mm}$ ) and the circular cross section of the impedance tube, a tapered shape adaptor was used. On the right hand side of the tube, a soundproof plunger is used as the rigid backing wall. The sealing for the plunger is ensured using a rubber seal. By moving the plunger along the longitudinal axis of the tube, it is possible to create an air cavity behind the sample. With an air cavity behind the perforated plate, the particle velocity is increased inside the pores at particular frequencies. This increase of velocity leads to a maximum of the visco-thermal losses. The maximum of absorption is obtained when the total reactance of the system is zero. Since each system formed by a sample and the cavity behind has different reactance (due to different open area ratio), to compare the results of all the samples at a given frequency, it is necessary to make use of different air cavity depth. The different air cavity depths considered in the measurements are given in Table 2 . Three $1 / 4^{\prime \prime}$ microphones are used to perform the signal detection. The first two microphones (micro 1 and 2 in Fig. 3) are used to calculate the surface impedance of the sample by the two-microphone standing waves method (see Ref. [18] for more details). A phase and amplitude calibration method is used to correct the transfer function between the measurement microphones. The distance between these microphones is $s=50 \mathrm{~mm}$. The distance between microphone 2 and the sample is about $l_{2}=110 \mathrm{~mm}$. The third microphone (reference micro in Fig. 3) is used to monitor the level of pressure at the sample surface.

Assuming the plane wave hypothesis, one can measure the pressures and velocities in any section of the tube using the twomicrophone method. See for instance Dalmont [19] for more details. The acoustic velocity on the panel surface is given by:

$u=j \frac{p_{1}}{Z_{0}} \frac{H \cos \left(k_{0} l_{1}\right)-\cos \left(k_{0} l_{2}\right)}{\sin \left(k_{0} s\right)}$,

where $l_{1}=s+l_{2}$ as in Fig. 3, $H$ is the ratio of pressures measured on microphones 1 and $2, k_{0}$ is the wave number, $p_{1}$ is the pressure on microphone 1 , and $l_{1}$ (resp. $l_{2}$ ) is the distance from microphone 1 (resp. 2) to the sample tested.

\section{Results discussion}

\subsection{Effects of holes interaction on nonlinear resistance}

Fig. $4 \mathrm{a}$ and $\mathrm{b}$ shows the normalized surface resistance of the samples as a function of the Reynolds number inside the apertures for (a) excitation frequency of $292 \mathrm{~Hz}$ and (b) excitation frequency of $506 \mathrm{~Hz}$. The computed value of $C_{2}$ is very close to 1 , which confirms similar observations reported in Refs. $[4,12]$ where it is proved that for high sound pressure levels, the dependence of the resistance on the velocity is linear. The first coefficient $C_{1}$ can then be considered as a slope and the dependence will be similar to Forchheimer's law for rigid porous media [21]. It is observed in both figures ( $a$ and $b$ ) that this slope decreases with a decrease of distance $b$. From these latter figures, as presented, the effect of holes interaction is combined to the effect of porosity. The decrease of the slope with an increase of the Reynolds numbers is then caused both by the increase of porosity and the HIE. As was also noticed by Melling [12], the influence of holes interaction can not be separated from the influence of porosity. An increase of the effect of holes interaction means a decrease of the distance between the holes and therefore an increase of the porosity. The effect caused only by the increase in porosity is hence difficult to evaluate. It is shown in other papers $[11,12,22]$ that the nonlinear regime slope tend to be inversely proportional to the porosity (mostly for high porosity samples). One can then clearly observe that for high sound pressure levels, the slope depends on the holes 
Table 2

Curve fitting coefficients for the nonlinear resistance of the samples. The air cavity depth is tuned so as to obtain the desired resonant frequencies of study for each sample.

\begin{tabular}{|c|c|c|c|c|c|c|c|c|}
\hline & \multicolumn{2}{|l|}{$C_{1}$} & \multicolumn{2}{|l|}{$\underline{C_{2}}$} & \multicolumn{2}{|l|}{$\underline{\delta}$} & \multicolumn{2}{|c|}{ Air cavity depth $(\mathrm{mm})$} \\
\hline & $292 \mathrm{~Hz}$ & $506 \mathrm{~Hz}$ & $292 \mathrm{~Hz}$ & $506 \mathrm{~Hz}$ & $292 \mathrm{~Hz}$ & $506 \mathrm{~Hz}$ & $292 \mathrm{~Hz}$ & $506 \mathrm{~Hz}$ \\
\hline Sample $1 \mathrm{~A}$ & $11 \times 10^{-3}$ & $4 \times 10^{-3}$ & 0.96 & 1 & 0.99 & 1.91 & 100 & 38 \\
\hline Sample $2 \mathrm{~A}$ & $2.5 \times 10^{-3}$ & $2.3 \times 10^{-3}$ & 0.98 & 0.98 & 0.85 & 3.06 & 180 & 75 \\
\hline Sample $3 A$ & $10^{-3}$ & $0.8 \times 10^{-3}$ & 0.98 & 0.99 & 4.09 & 5.96 & 200 & 142 \\
\hline Sample $4 \mathrm{~A}$ & - & $0.7 \times 10^{-3}$ & - & 0.99 & - & 5.63 & - & 150 \\
\hline
\end{tabular}
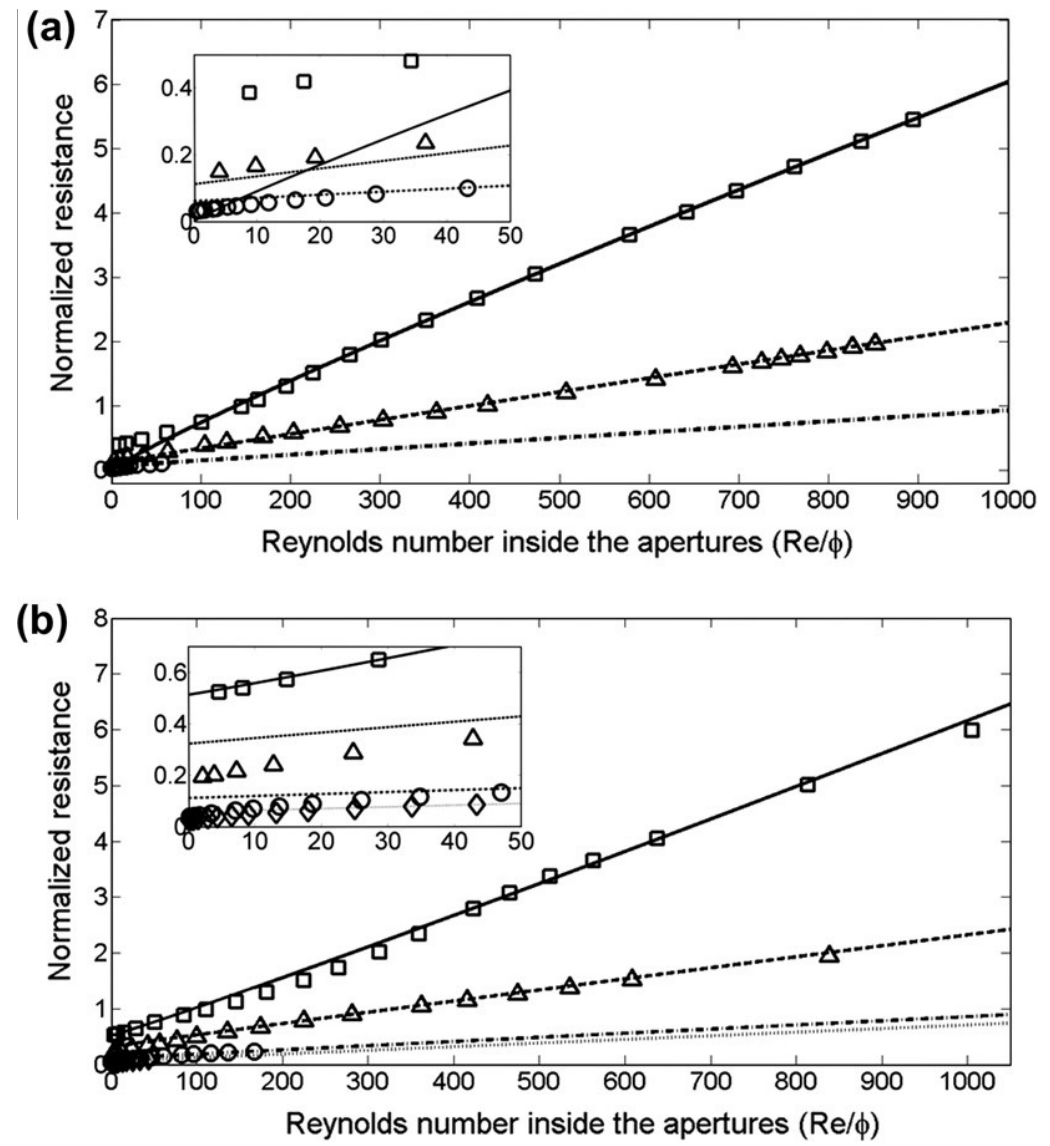

Fig. 4. Normalized surface resistance as a function of the Reynolds number inside the apertures: (a) Excitation frequency of $292 \mathrm{~Hz}$; (b) Excitation frequency of $506 \mathrm{~Hz}$. Symbols are for measurements and lines for simulations. $\square$ and solid line: sample $1 \mathrm{~A}(b=12 \mathrm{~mm}) ; \Delta$ and dashed line: sample $2 \mathrm{~A}(b=08 \mathrm{~mm}) ; \bigcirc$ and dashed-dotted line: sample $3 \mathrm{~A}(b=3.5 \mathrm{~mm}) ; \diamond$ and dotted line: sample $4 \mathrm{~A}(b=2.6 \mathrm{~mm})$.

separation and decreases with the increase of Reynolds number inside the apertures due to interaction between them. A convenient explanation of this observation is that as distance $b$ decreases, the shear region around each hole is shared with the shear region around the nearby holes. The total acoustic resistance of the system is mainly caused by the apertures resistance and the vorticities induced resistance at the inlet and the outlet of the apertures. Since the total shear region is reduced for interacting holes, this causes a reduction in the radiation resistance. This results in a reduction of the total acoustic resistance. According to Table 2, the dependence of these nonlinear coefficients on frequency tends to be less sensitive to a decrease of $b$ (results carried out for $292 \mathrm{~Hz}$ and $506 \mathrm{~Hz}$ ).

\subsection{Effects of holes interaction on nonlinear reactance}

Fig. 5a and $\mathrm{b}$ shows the experimental and predictions of the normalized surface reactance of the samples as a function of the Reynolds number inside the apertures. For each sample tested, the same air cavity depth is kept throughout the whole range of Reynolds number studied. The observed changes in the reactance are relatively very weak compared to that of the resistance. As it was already noted by Melling [12], with the increase of sound pressure levels, the reactance decreases. For both interacting and not interacting holes, this decrease is not a consequence of Forchheimer's law but rather a transfer of part of the kinetic energy in the sound field around the aperture into turbulent motion that breaks away from the aperture. This is a typical nonlinear behavior of the reactance of thin plates whenever turbulence is reached (see Ref. [4]). Since this behavior is less likely to take place for the cases of closely separated holes (samples $3 \mathrm{~A}$ and $4 \mathrm{~A}$ ), it is observed that with the decrease of $b$, the decrease in reactance is less significant. Due to the high velocity of the fluid particles along the perforation axis, a drag (squeeze) effect occurs on the particle on the outer (inner) side of the baffle. This effect is reduced when the interaction effect occurs as the region of influence is shared. Nevertheless, as stated by Ingard [4], there is a close tie between the nonlinear resistance and the reactance, the loss in mass reactance results in 

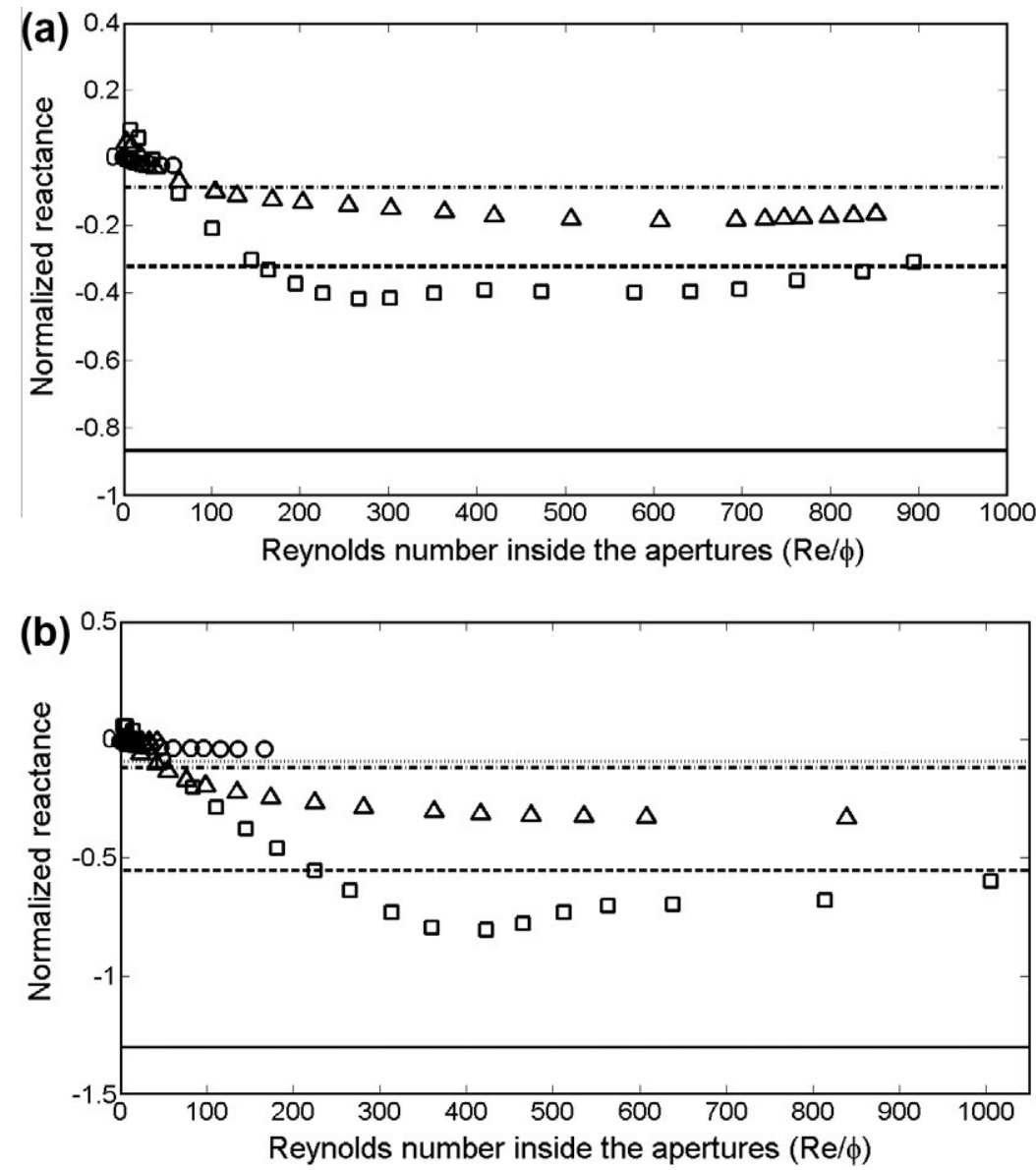

Fig. 5. Experimental results of the normalized surface reactance as a function of the Reynolds number inside the apertures: (a) Excitation frequency of $292 \mathrm{~Hz}$; (b) Excitation frequency of $506 \mathrm{~Hz}$. Symbols are for measurements and lines for simulations. $\square$ and solid line: sample $1 \mathrm{~A}(b=12 \mathrm{~mm}) ; \triangle$ and dashed line: sample $2 \mathrm{~A}(b=08 \mathrm{~mm}) ; \bigcirc$ and dashed-dotted line: sample $3 \mathrm{~A}(b=3.5 \mathrm{~mm}) ; \diamond$ and dotted line: sample $4 \mathrm{~A}(b=2.6 \mathrm{~mm})$.

the increase in the resistance. The model fails to agree with the experimental results. In particular, the decrease of reactance is not predicted by the model. This result was expected since the dependence of reactance on the velocity is neglected in the model. As was first noticed by Melling [12], then later on by Peat [23,24], the derivation of Fok's function follows explicitely from a potential flow analysis for the reactance only and, from a physical viewpoint, one would not expect an identical interaction effect for resistance. In general, the reactance effect variations result in a shift of the resonance frequency. Thus, for these samples, with an increase of the Reynolds number inside the perforations, the resonance frequency is observed to shift towards the high frequency range by about $50 \mathrm{~Hz}(b=12 \mathrm{~mm}), 30 \mathrm{~Hz} \quad(b=8 \mathrm{~mm}), 11 \mathrm{~Hz} \quad(b=3.5$ $\mathrm{mm})$ and $6 \mathrm{~Hz}(b=2.6 \mathrm{~mm})$. Unlike Maa [20], most authors $[12,3,11]$ do not consider this shift of resonance frequency.

\subsection{Effects of holes interaction on absorption coefficient peak value}

Fig. $6 \mathrm{a}$ and $\mathrm{b}$ shows the experimental and predictions of the maximum of the absorption coefficient as a function of the Reynolds number inside the apertures. The maximum of absorption coefficient is obtained when the imaginary part of the normalized surface impedance equals to zero $\left(\operatorname{Imag}\left\{z_{S}\right\}=0\right)$. The simulations and the experimental results are in very good agreement for both frequencies of excitation. Depending on the sample characteristics (particularly on the hole diameters and the open area ratio), the absorption coefficient achieves its maximum value of 1 and then decreases with the increase of
Reynolds number (Refs. [21,22]). The critical Reynolds number is defined as a value at which absorption coefficient attains its maximum. These values are predicted and measured for samples $1 \mathrm{~A}$ and $2 \mathrm{~A}$. The critical Reynolds numbers for samples $3 \mathrm{~A}$ and $4 \mathrm{~A}$ fall outside the range achieved in experiments. If $b$ and $\phi$ are optimized, the HIE can contribute to absorption coefficient increase under medium and high sound pressure levels. In the following section an expression for optimizing this critical Reynolds number is derived.

\subsection{Critical Reynolds number and the distance between the apertures}

In this section, an expression for the critical Reynolds number as a function of the center-to-center distance between the perforations is derived. This expression is valid only for high sound pressure levels regime. The presented critical Reynolds number $\operatorname{Re}_{C}$ is the value of the Reynolds number for which the maximum absorption peak reaches its extremum. Beyond $\operatorname{Re}_{c}$, the peak of absorption (maximum of absorption with respect to frequency) decreases with an increase of the Reynolds number. It can easily be shown that the maximum of absorption coefficient $\alpha_{M}$ is obtained for $\operatorname{Imag}\left\{z_{S}\right\}=0$

$\alpha_{M}=\frac{4 \operatorname{Real}\left\{z_{S}\right\}}{\left(1+\operatorname{Real}\left\{z_{S}\right\}\right)^{2}}$,

where $\operatorname{Real}\left\{z_{S}\right\}$ is the normalized surface resistance. Under high sound pressure levels, $\operatorname{Real}\left\{z_{s}\right\}$ is given by Eq. (12) and a maximum of absorption coefficient of 1 is obtained for $\operatorname{Real}\left\{z_{S}\right\}=1$ that is 

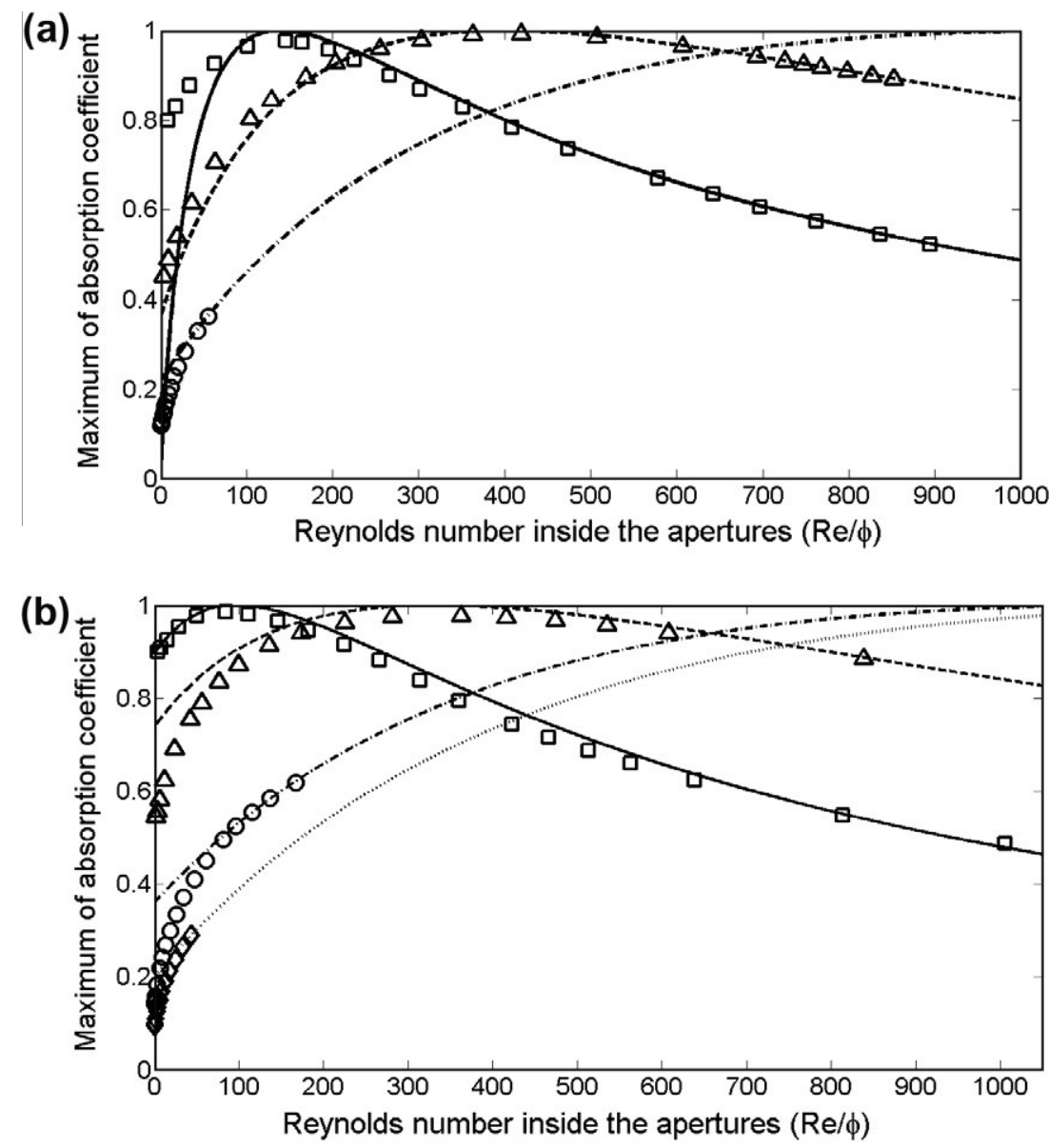

Fig. 6. Maximum of absorption coefficient as a function of the Reynolds number inside the apertures: (a) Excitation frequency of $292 \mathrm{~Hz}$; (b) Excitation frequency of $506 \mathrm{~Hz}$ Symbols are for measurements and lines for simulations. $\square$ and solid line: sample $1 \mathrm{~A}(b=12 \mathrm{~mm}) ; \Delta$ and dashed line: sample $2 \mathrm{~A}(b=08 \mathrm{~mm}) ; \bigcirc$ and dashed-dotted line: sample $3 \mathrm{~A}(b=3.5 \mathrm{~mm}) ; \diamond$ and dotted line: sample $4 \mathrm{~A}(b=2.6 \mathrm{~mm})$.

$C_{1}\left(\operatorname{Re}_{C}\right)^{C_{2}}=1$.

If $C_{2}$ is approximately 1 as was proven earlier, then the critical Reynolds number is given by

$\operatorname{Re}_{C}=\sqrt{\frac{1}{C_{1}}}$

As was shown in [22], where Forchheimer's law was used to model the nonlinear resistance, $C_{1}$ can be approximated as

$C_{1}=\frac{K}{\phi}$,

where $K$ is a constant equal to $2.2 \times 10^{-3}$ for the aluminum sample. The value of $K$ is dependent on the shape of the aperture (see Ref. [22]). According to Everest and Pohlmann [25], for a triangular lattice arrangements of the apertures, the open area ratio is given by

$\phi=0.906 \frac{d^{2}}{b^{2}}$.

Substituting Eq. (23) into Eq. (22) and the resulting equation into Eq. (21), one obtains

$\operatorname{Re}_{C}=\frac{d}{b} \sqrt{\frac{0.906}{K}}$

This is an important result for the design of optimal perforated plates subjected to high sound pressure levels. It states that the critical Reynolds number is directly proportional to the diameter of the holes and inversely proportional to the distance between the perforations. Eq. (24) is confirmed by the experimental results for sample $1 \mathrm{~A}$ and not for sample $2 \mathrm{~A}$. For sample $2 \mathrm{~A}$, the disagreement between the data and the predictions could be attributed to the fact that some holes were slightly tapered during the fabrication process. For samples $3 \mathrm{~A}$ and $4 \mathrm{~A}$, the critical Reynolds number values are outside the range achieved in experiments. As stated by Tayong et al. [22], all the parameters having an influence on the value of $K$ are not fully understood, both the edges shape and the type of material seem to affect the value of $K$ as was shown in this latter paper.

\section{Conclusion}

An experimental investigation of the holes interaction effect (HIE) of air-cavity-backed perforated plates under high and medium sound levels was carried out in this paper. The study was focused on this effect on the acoustic impedance and absorption coefficient. From the theoretical and experimental work presented, the following results were obtained:

- The linear variation of the acoustic resistance with the Reynolds number inside the aperture depends on the center-to-center distance of the holes. The stronger the HIE, the more the slope decreases. A fitting of this slope provides two adjustable coefficients suitable for modeling the system. 
- The stronger the HIE, the smaller the variation of the acoustic reactance with the Reynolds number. However, it is noticed that this variation remains negligible compared to the acoustic resistance variation.

- Since the HIE modifies both the acoustic resistance and reactance, it also modifies the maximum of the absorption coefficient. A critical Reynolds number, defined as the Reynolds number value after which the maximum of absorption coefficient starts to decrease, was introduced. Both the prediction and the experiment revealed that a decrease of the center-tocenter distance results into a shift (towards higher values) of this critical Reynolds number.

- An expression that relates the critical Reynolds number and the center-to-center distance was derived. This expression can lead to the design of optimal perforated plates (higher absorption coefficient amplitude for large Reynolds number ranges).

\section{Acknowledgement}

The support grant for this work was provided by the Conseil Régional de Bourgogne.

\section{References}

[1] Allard JF. Propagation of sound in porous media. London: Elsevier; 1993

[2] Maa DY. Potential of micro-perforated panel absorber. Journal of the Acoustical Society of America 1998;104:2861-6.

[3] Hersh AS, Walker BE, Celano JW. Helmholtz resonator impedance model, Part I nonlinear behavior. American Institute of Aeronautics and Astronautics 2003;41(5):795-808

[4] Ingard U. On the theory and design of acoustic resonators. Journal of the Acoustical Society of America 1953:25(6):1037-61.

[5] Atalla N, Sgard F. Modeling of perforated plates and screens using rigid frame porous models. Journal of Sound and Vibration 2007;303:195-208.

[6] Fok VA. Doklady akademii nauk. SSSR (31); 1941 (in Russian). Alternatively, see the next reference.
[7] Rzhevkin SN. A course of lectures on the theory of sound. London: Pergamon Press; 1963.

[8] Ingard U, Ising H. Acoustic nonlinearity of an orifice. Journal of the Acoustical Society of America 1967;42:6-17.

[9] Cummings A. Acoustic nonlinearity and power losses at orifices. AIAA 1984;22:786-92.

[10] Cummings A. Transient and multiple frequency sound transmission through perforated plates at high amplitudes. Journal of the Acoustical Society of America 1986;79:942-51.

[11] Kraft RE. Acoustic treatment design scaling methods. Numerical simulation of the nonlinear acoustic impedance of a perforated plate single-degree-of freedom resonator using a time-domain finite difference method, US. NASA Report No. CR-1999-209120, vol. 4; 1999.

[12] Melling TH. The acoustic impedance of perforates at medium and high sound pressure levels. Journal of Sound and Vibration 1973;29(1):1-65.

[13] Randeberg RT. Perforated panel absorbers with viscous energy dissipation enhanced by orifice design. PhD thesis, Trondheim; 2000. p. 15-18.

[14] Lee S-H, Ih J-G, Peat KS. A model of acoustic impedance of perforated plates with bias flow considering the interaction effect. Journal of Sound and Vibration 2007;303(3-5):741-52.

[15] Tayong R, Dupont T, Leclaire P. Hole interaction effects under high and medium sound intensities for micro-perforated panels design. In: 10th French congress of acoustics, Lyon; April 2010.

[16] Rayleigh L. Theory of sound. London: Macmillan; 1940.

[17] Maa DY, Liu K. Nonlinear standing waves: theory and experiments. Journal of the Acoustical Society of America 1995;98(5):2753-63.

[18] Chung JY, Blaser DA. Transfer function method of measuring in-duct acoustic properties. Part I: Theory. Journal of the Acoustical Society of America 1980;68(3):907-13

[19] Dalmont J-P. Acoustic impedance measurement. Part I: A review. Journal of Sound and Vibration 2001;243(3):441-59.

[20] Maa DY. Micro-perforated panel at high sound intensity. Inter noise, Yokohama Japan; 1994. p. 1511-1514.

21] Umnova O, Attenborough K, Standley E, Cummings A. Behavior of rigid-porous layers at high levels of continuous acoustic excitation: theory and experiment. Journal of the Acoustical Society of America 2003;114(3):1346-56.

[22] Tayong R, Dupont T, Leclaire P. On the variations of acoustic absorption peak with particle velocity in micro-perforated panels at high level of excitation. Journal of the Acoustical Society of America 2010;127(5):2875-83.

[23] Peat KS. End correction at the interface between a plain and a perforated pipe. Journal of Sound and Vibration 2009;319(3-5):1097-106.

[24] Peat KS. Acoustic impedance at the interface between a plain and a perforated pipe. Journal of Sound and Vibration 2010;329(14):2884-94.

[25] Everest FA, Pohlmann KC. Master handbook of acoustics. New York: McGraw Hill; 2009. 